\title{
A parameter-free optical potential for the heavy-ion elastic scattering process
}

\author{
M. A. G. Alvarez, L. C. Chamon, M. S. Hussein, D. Pereira, L. R. Gasques, E. S. Rossi Jr., C. P. Silva. \\ Departamento de Física Nuclear, Instituto de Física da Universidade de São Paulo, \\ Caixa Postal 66318, 05315-970, São Paulo, SP, Brazil.
}

\begin{abstract}
Thirty elastic scattering angular distributions for seven heavy-ion systems, in wide energy ranges, have been studied with the aim of systematizing the optical potential, real and imaginary parts, in a global way. The framework is: i) an extensive systematization of nuclear densities, ii) the energy dependence of the bare potential accounted by a model based on the nonlocal nature of the interaction, and iii) the real and imaginary parts of the optical potential assumed to have the same radial shape.
\end{abstract}

PACS: 24.10.Ht, 25.70.-z, 25.70.Bc

Keywords: Heavy-ion optical potential. Heavy-ion elastic scattering.

Elastic scattering is the simplest and most direct process involved in a nuclear reaction, and it can be used as the starting point to understand more complicated reaction channels. Over the last decades, different models were used for the real and imaginary parts of the optical potential to reproduce a large number of elastic scattering data involving heavy-ion systems. The optical model analysis using the conventional Woods-Saxon (WS) shape for the real and imaginary parts of the potential, although far from being fundamental, has presented the best means for the reproduction of the elastic scattering angular distributions, with six free parameters used in the data fits. However, there are problems in terms of obtaining a simple model for systematizing the WS optical potential parameters, in order to take into account the energy dependence, refractive effects in light heavy-ion systems, exotic nuclei systems etc. Particularly, for some systems the variation with the bombarding energy requires arbitrarily different sets of parameters, with different shapes and strengths for the potential. This arbitrariness calls for a more realistic model for the optical potential, which has been accomplished to a large extent by our work [1,2,3, 4. 5 on the nonlocal model for the real part of the nucleus-nucleus interaction. The central idea of the present work is to perform a further test of consistency of this model for the real part of the interaction, by using a very simple form to describe the imaginary part of the optical potential, and avoiding as much as possible the use of free parameters in accounting to the data.

A previous investigation [6, 6 ,8] to identify similarities between the real and imaginary parts of the potential has been performed by considering the realistic Lax-type interaction, which has provided satisfactory fits of elastic scattering data at intermediate energies [6.7]. The Lax interaction [9] (Eq. 1) is the optical limit of the Glauber high-energyapproximation [10,11], and it is essentially the zero-range double-folding potential used for both the real and imaginary parts:

$$
U(R)=-\frac{1}{2} \hbar v \int(\alpha+i) \sigma_{T}^{N N} \rho_{T}\left(\overrightarrow{r^{\prime}}\right) \rho_{P}\left(\vec{R}-\overrightarrow{r^{\prime}}\right) d \overrightarrow{r^{\prime}}
$$

where $v$ is the relative velocity between the nuclei, $\sigma_{T}^{N N}$ is a spin-isospin-averaged total nucleon-nucleon cross section, $\rho_{P}$ and $\rho_{T}$ are the projectile and target nuclear densities, and $\alpha$ is a known energy-dependent quantity that determines the real part of the nucleon-nucleon forward elastic amplitude $f_{N N}(E, 0)$. Eq. (1) is obtained from the optical theorem applied to $f_{N N}(E, 0)$. The Lax-type interaction is not valid for low energies where collective reaction processes are important. Thus, the parameter-free description of low energy data is an open question in the determination of a fundamental potential. However, the procedure of using the same radial shape for the real and the imaginary parts of the potential has successfully been used in the present work.

In [1, 2, 3, 4, 5], we have developed another realistic model for the heavy-ion bare interaction, which takes into account the Pauli nonlocality involving the exchange of nucleons between the target and projectile. This model has presented the same validity at low and high energies, and has already been tested for a large number of systems [1, 2, 3, 5, 12, 13, 14, 15]. Within the nonlocal model, the bare interaction $V_{N}$ is connected with the folding potential $V_{F}$ through [5]

$$
V_{N}(R, E) \approx V_{F}(R) e^{-4 v^{2} / c^{2}}
$$

where $\mathrm{c}$ is the speed of light and $v$ is the local relative velocity between the two nuclei,

$$
v^{2}(R, E)=\frac{2}{\mu}\left[E-V_{C}(R)-V_{N}(R ; E)\right]
$$


The folding potential (Eq. 4) can be obtained in two different ways (57): i) using the nucleon distributions of the nuclei and an appropriate form for the nucleon-nucleon interaction, and ii) using the matter distributions of the nuclei with a zero-range approach for $v(\vec{r})$. By matter distribution we mean taking into account the finite size of the nucleon. Both alternatives are equivalent in describing the heavy-ion nuclear potential [5], and in the present work we have adopted the zero-range approach.

$$
V_{F}(R)=\int \rho_{1}\left(r_{1}\right) \rho_{2}\left(r_{2}\right) v\left(\vec{R}-\overrightarrow{r_{1}}+\overrightarrow{r_{2}}\right) d \overrightarrow{r_{1}} d \overrightarrow{r_{2}}
$$

For the Coulomb interaction, $V_{C}$, we have used the expression for the double-sharp cutoff Coulomb potential [16]. This procedure is important in calculating cross sections at intermediate energies, where the internal region of the interaction is probed.

With the aim of providing a global description of the nuclear interaction, a systematization of nuclear densities has been proposed in Ref. [5], based on an extensive study involving charge distributions extracted from electron scattering experiments and theoretical densities calculated through the Dirac-Hartree-Bogoliubov model. In that study, we have adopted the two-parameter Fermi $(2 \mathrm{pF})$ distribution to describe the nuclear densities. The radii of the $2 \mathrm{pF}$ distributions are well described by

$$
R_{0}=1.31 A^{1 / 3}-0.84 \mathrm{fm},
$$

where $A$ is the number of nucleons of the nucleus. The matter densities present an average diffuseness value $a=$ $0.56 \mathrm{fm}$. Owing to specific nuclear structure effects (single particle and/or collective), the parameters $R_{0}$ and $a$ show small variations around the corresponding average values throughout the periodic table. However, as far as the nuclear potential is concerned, the effects of the structure of the nuclei are mostly present at the surface and mainly related only to the diffuseness parameter [5]. This systematization of the nuclear distributions is essential to obtain a parameter-free interaction, since the folding potential depends on the densities of the partners in the collision. Within this context, an extensive systematization of optical potential strengths extracted from heavy-ion elastic scattering data analyses at low and intermediate energies was performed [5], and the experimental potential strengths have been described within $25 \%$ precision.

As mentioned above, an important point that stands out in obtaining a description of the optical potential in a global way is the difficulty encountered in describing the imaginary part of the interaction within a simple model. A fully microscopic description based on the Feshbach theory is very difficult, and basically out of reach at low energies where collective as well as single particle excitations are involved in the scattering process. In previous works involving elastic scattering data fits [2,3, 12, 13, 14, 15], we have already used the nonlocal model for the real part of the interaction, and adopted a more modest procedure for the imaginary part by assuming two different models: WS with three free parameters, which has presented an excellent description of the data; and the parameter-free Lax-type approximation, which is based on a more fundamental theory but has not been used to describe low energy data. Motivated by the concept from the Lax approximation of using similar shapes for the real and imaginary parts of the potential, in this work we have extended Eq. (2), developed for the real part of the interaction, to the imaginary part of the potential, by simply multiplying it by $N_{i}$, where $N_{i}$ is a number to be fixed by adjusting the data.

$$
W(R, E)=N_{i} V_{N}(R, E)
$$

We have chosen the ${ }^{12} \mathrm{C}+{ }^{12} \mathrm{C},{ }^{16} \mathrm{O},{ }^{40} \mathrm{Ca},{ }^{90} \mathrm{Zr},{ }^{208} \mathrm{~Pb} ;{ }^{16} \mathrm{O}+{ }^{208} \mathrm{~Pb}$ and ${ }^{40} \mathrm{Ar}+{ }^{208} \mathrm{~Pb}$ systems as test cases due to the extensive experimental data available [17, 18, 19, 20,21,22, 23,24,25,26] over wide energy ranges, and principally because the special refractive characteristics involving some of these systems which makes them more sensitive to the real part of the interaction. In Figures (1) to (7) the solid lines correspond to the best data fits obtained by searching the $N_{i}$ parameter. We have opted for keeping the average density diffuseness value $a=0.56 \mathrm{fm}$ in the calculations, even though we could improve the quality of the data fits by allowing the diffuseness to be a free parameter. As one can observe (see Fig. 8), the $N_{i}$ parameter is approximately system-independent, with an average value $N_{i}=0.78$. Good elastic scattering cross section predictions are obtained using this average value for the whole data set (see the dotted lines in Figs. 1 to 7 ).

In summary, using the procedure described above we have obtained a good description of the whole data set, which has further validated our assumption for the real part of the interaction: the nonlocal model. We have also assumed a very simple model for the imaginary part of the potential, with only one, system- and energy-independent, free parameter: the average value for $N_{i} \approx 0.8$. In fact, the details of the imaginary part of the interaction seems not to be of much importance to the data fit. For example, in Fig. 7 (bottom) quite different values for $N_{i}\left(N_{i}=0.44\right.$ and $\left.N_{i}=0.78\right)$ provide very similar predictions for the elastic scattering cross sections. The same behavior can be 
observed for the other systems (see Figs. 1 to 7 ). At a first glance this result seems to be surprising, but upon a second thought one does expect that the data are more sensitive to the real part of the potential, which determines the quantal transmission through the $\ell$-dependent barriers. In order to confirm this point, we have compared (see table 1) the reaction cross sections resulting from our optical model (OM) calculations (using the average value for $N_{i}$ ) with those from the geometrical limit of the barrier penetration model (Eq. 7)

$$
\sigma_{B P}=\pi R_{B}^{2}\left(1-V_{B} / E\right)
$$

In most cases both forms of calculating the reaction cross section agree within about $20 \%$ precision. However, the values obtained from the OM calculations are more realistic. Indeed the reaction cross section values obtained with our OM calculations are very similar (see table 1) to those obtained through different methods in earlier works [6, 17, 18, 22, 23, 24, 27, 28, 29, 30, 31, 32]. Our results suggest using the present parameter-free model to get reliable estimates for heavy-ion elastic scattering and reaction cross sections. Extension of our findings to halo-nuclei is being pursued and will be presented elsewhere.

This work was partially supported by Financiadora de Estudos e Projetos (FINEP), Conselho Nacional de Desenvolvimento Científico e Tecnológico $(\mathrm{CNPq})$ and Fundação de Amparo à Pesquisa do Estado de São Paulo (FAPESP), under contract number 1998/11401-4.

[1] M. A. Candido Ribeiro, L. C. Chamon, D. Pereira, M. S. Hussein and D. Galetti, Phys. Rev. Lett. 78, 3270 (1997).

[2] L. C. Chamon, D. Pereira, M. S. Hussein, M. A. Candido Ribeiro and D. Galetti, Phys. Rev. Lett. 79, 5218 (1997).

[3] L. C. Chamon, D. Pereira, and M. S. Hussein, Phys. Rev. C58, 576 (1998).

[4] D. Galetti, S. S. Mizrahi, L. C. Chamon, D. Pereira, M. S. Hussein and M. A. Candido Ribeiro, Phys. Rev. 58, 1627 (1998).

[5] L. C. Chamon, B. V. Carlson, L. R. Gasques, D. Pereira, C. De Conti, M. A. G. Alvarez, M. S. Hussein, M. A. Candido Ribeiro, E. S. Rossi Jr. and C. P. Silva, Phys. Rev. C66, (2002) 014610.

[6] M. E. Brandan, H. Chehime and K. W. McVoy, Phys. Rev. C55, 1353 (1997).

[7] S. K. Charagi and S. K. Gupta, Phys. Rev C56, 1171 (1997).

[8] M. E. Brandan, K. W. Mc Voy, Phys. Rev. C55, 1362 (1997).

[9] see, e.g., M. S. Hussein, R. A. Rego and C. A. Bertulani, Phys. Rep. 201, 279 (1991).

[10] R. J. Glauber, in Lectures in Theoretical Physics, edited by W. E. Brittin and L. G. Dunham (Wiley-Interscience, New York, 1959), Vol. 1, p. 315; in High Energy Physics and Nuclear Structure, edited by G. Alexander (Wiley, New York, 1967), p 311; in High Energy Physics and Nuclear Structure (Plenum, New York, 1970), p. 207.

[11] W. Czyz and L. C. Maximon, Ann. Phys. (N. Y.) 52, 59 (1969).

[12] M. A. G. Alvarez, L. C. Chamon, D. Pereira, E. S. Rossi Jr., C. P. Silva, L. R. Gasques, H. Dias and M. O. Roos, Nucl. Phys. A656, 187 (1999).

[13] M. A. G. Alvarez, E. S. Rossi Jr., C. P. Silva, L. R. Gasques, L. C. Chamon, D. Pereira, M. N. Rao, B. V. Carlson, C. De Conti, R. M. Anjos, P. R. S. Gomes, J. Lubian, S. Kailas, A. Chatterjee and P. Singh, Phys. Rev. C65, 014602 (2002).

[14] L. R. Gasques, L. C. Chamon, C. P. Silva, D. Pereira, M. A. G. Alvarez, E. S. Rossi Jr., V. P. Likhachev, B. V. Carlson and C. De Conti. Phys. Rev. C65, (2002) 044314.

[15] E. S. Rossi Jr., D. Pereira, L. C. Chamon, C. P. Silva, M. A. G. Alvarez, L. R. Gasques, J. Lubian, B. V. Carlson and C. De Conti, Nuc. Phys. A707, 325 (2002).

[16] R. M. Devries and M. R. Clover, Nucl. Phys., A243, 528 (1975).

[17] D. T. Khoa, W. von Oertzen and H. G. Bohlen, Phys. Rev. C49, 1652 (1994).

[18] A. A. Ogloblin, Y. A. Glukhov, V. Trzaska, A. S. Demyanova, S. A. Goncharov, R. Julin, S. V. Klebnikov, M. Mutterer, M. V. Rozhkov, V. P. Rudakov, G. P. Tiorin, D. T. Khoa and G. R. Satchler. Phys. Rev. C62, 44601 (2000).

[19] V. Chiste, R. Lichtenthaler, A. C. C. Villari and L. C. Gomes, Phys. Rev. C54, 784 (1996).

[20] J. B. Ball, C. B. Fulner, E. E. Gross, M. L. Halbert, D. C. Hensley, C. A. Ludemann, M. J. Saltmarsh and G. R. Satchler. Nucl. Phys. A252, 208 (1975).

[21] Y. T. Oganessian and Y. E. Penionzhkevich, V. I. Man'Ko and V. N. Polyansky, Nucl. Phys. A303, 259 (1978).

[22] C. C. Sahm, T. Murakami, J. G. Cramer, A. J. Lazzarini, D. D.Leach, D. R. Tieger, R. A. Loveman, W. G. Lynch, M. B. Tsang and J. Van der Plicht. Phys. Rev. C34, 2165 (1986).

[23] P. Roussel-Chomaz, N. Alamanos, F. Auger, J. Barrette, B. Berthier, B. Fernandez and L. Papineau, Nucl. Phys. A477, (1988) 345.

[24] J. Y. Hostachy, M. Buenerd, J. Chauvin, D. Lebrun, Ph. Martin, J. C. Lugol, L. Papineau, P. Roussel, N. Alamanos, J. Arviex, and C. Cerruti, Nucl. Phys. A490, 441 (1998). 
[25] M. Buenerd, J. Pinston, J. Cole, C. Guet, D. Lebrun, J. M. Loiseaux, P. Martin, E. Monnand, J. Mougey, H. Nifenecker, R. Ost, P. Perrin, Ch. Ristori, P. de Saintgnon, F. Schussler, L. Carlen, H. A. Gustafsson, B. Jakobsson, T. Johansson, G. Jonsson, J. Krumlinde, I. Otterlund, H. Ryde, B. Schroder, G. Tibell, J. B. Bondorf, and O. B. Nielsen, Phys. Lett. 102B, 242 (1981).

[26] M. Buenerd, A. Lounis, J. Chauvin, D. Lebrun, P. Martin, G. Duhamel, J. C. Gondrand, and P. de Saintgnon, Nucl. Phys. A424, 313 (1984)

[27] N. Ohtsuka, R. Linden and Amand Faesler, Phys. Lett. 199B, 330 (1987).

[28] S. K. Charagi, Phys. Rev. C51, 3521 (1995).

[29] C. Olmer, M. Mermaz, M. Buerned, C. K. Gelbke, D. L. Hendrie, J. Mahoney, D. K. Scott, M. H. Macfarlane and S. C. Pieper, Phys. Rev. C18, 205 (1978).

[30] W. Treu, H. Frohlich, W. Galster, P. Duck and H. Voit, Phys. Rev. C22, 2462 (1980).

[31] H. G. Bohlen, M. R. Clover, G. Ingold, H. Lettan and W. von Oertzen, Z. Phys. A308, 121 (1982).

[32] S. Kox, A. Gamp, C. Perrin, J. Arvieux, R. Bertholet, J. F. Bruandet, M. Buenerd, R. Cherkaoui, A. J. Cole, Y. El-Masri, N. Longequeue, J. Menet, F. Merchez and J. B. Viano, Phys. Rev. C35, 1678 (1987). 
Table 1: The optical model $\left(\sigma_{O M}\right)$ and geometrical $\left(\sigma_{B P}\right)$ reaction cross sections obtained in this work for several systems and bombarding energies. The table also presents the values (and corresponding references) for the reaction cross sections $\left(\sigma_{R}\right)$ obtained through different methods in earlier works.

\begin{tabular}{|c|c|c|c|c|c|}
\hline System & $E_{L a b}(\mathrm{MeV}$ & $\sigma_{O M}(\mathrm{mb})$ & $\sigma_{B P}(\mathrm{mb})$ & $\sigma_{R}(\mathrm{mb})$ & Ref. \\
\hline \multirow{5}{*}{${ }^{12} \mathrm{C}+{ }^{12} \mathrm{C}$} & 16 & 542 & 508 & 600 & 30 \\
\hline & 112 & 1477 & 1759 & 1444 & 32 \\
\hline & 300 & 1374 & 1834 & 1296 & 31 \\
\hline & 1016 & 1055 & 1680 & 1000 & 24 \\
\hline & 1449 & 910 & 1560 & 907 & 24 \\
\hline \multirow{4}{*}{${ }^{16} \mathrm{O}+{ }^{12} \mathrm{C}$} & 21 & 209 & 167 & - & - \\
\hline & 260 & 1560 & 1877 & 1481 & 18 \\
\hline & 608 & 1420 & 1881 & 1374 & 18 \\
\hline & 1503 & 1143 & 1716 & 1136 & 23 \\
\hline \multirow{3}{*}{${ }^{12} \mathrm{C}+{ }^{40} \mathrm{Ca}$} & 180 & 2022 & 2083 & 2165 & 22 \\
\hline & 300 & 2015 & 2168 & 2030 & 22 \\
\hline & 420 & 1969 & 2180 & 2000 & 22 \\
\hline \multirow{4}{*}{${ }^{12} \mathrm{C}+{ }^{90} \mathrm{Zr}$} & 120 & 2310 & 2113 & 2219 & 22 \\
\hline & 180 & 2540 & 2406 & 2297 & 22 \\
\hline & 300 & 2652 & 2610 & 2415 & 22 \\
\hline & 420 & 2650 & 2670 & 2840 & 22 \\
\hline \multirow{8}{*}{${ }^{12} \mathrm{C}+{ }^{208} \mathrm{~Pb}$} & 96 & 1854 & 1498 & 1791 & 28 \\
\hline & 116 & 2323 & 1944 & 2235 & 28 \\
\hline & 300 & 3205 & 3013 & 3300 & 22 \\
\hline & 420 & 3611 & 3393 & 3561 & 22 \\
\hline & 480 & 3634 & 3441 & - & - \\
\hline & 1030 & 3560 & 3501 & - & - \\
\hline & 1449 & 3344 & 3331 & 3136 & 6 \\
\hline & 2400 & 2905 & 2882 & 2960 & 27 \\
\hline \multirow{4}{*}{${ }^{16} \mathrm{O}+{ }^{208} \mathrm{~Pb}$} & 129.5 & 1993 & 1553 & 2023 & 28 \\
\hline & 192 & 2878 & 2408 & 2847 & 28 \\
\hline & 312.6 & 3504 & 3067 & 3432 & 29 \\
\hline & 1500 & 3708 & 3530 & 3485 & 23 \\
\hline \multirow[t]{2}{*}{${ }^{40} \mathrm{Ar}+{ }^{208} \mathrm{~Pb}$} & 302 & 2476 & 1740 & - & - \\
\hline & 1760 & 4783 & 4059 & - & - \\
\hline
\end{tabular}




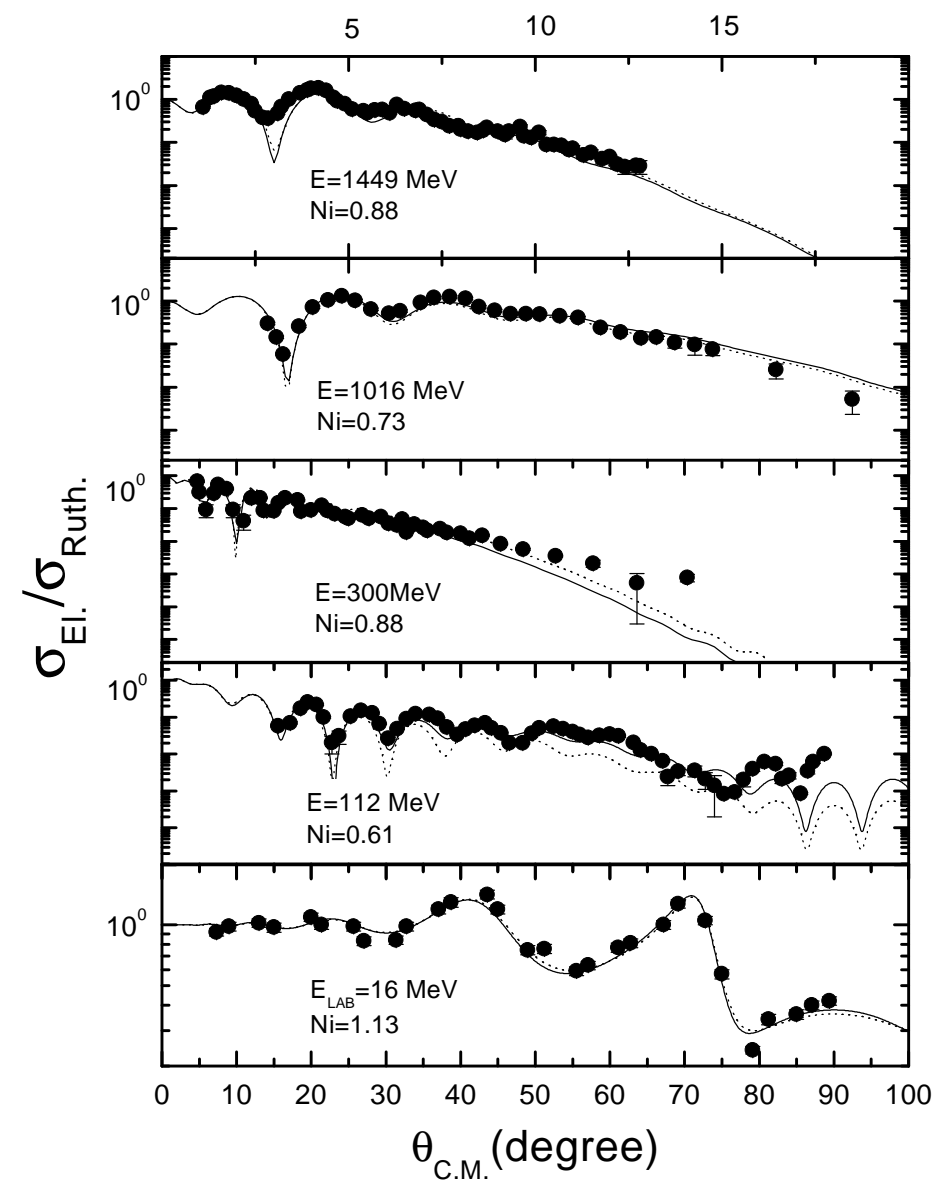

FIG. 1. Elastic scattering angular distributions for the ${ }^{12} \mathrm{C}+{ }^{12} \mathrm{C}$ system in several bombarding energies. The solid lines correspond to the best fit using the same radial shape for both the real and imaginary parts of the optical potential, with the $N_{i}$ parameter searched for the best data fits. The dotted lines correspond to the predictions obtained with the average value $N_{i}=0.78$ (see text for details). 


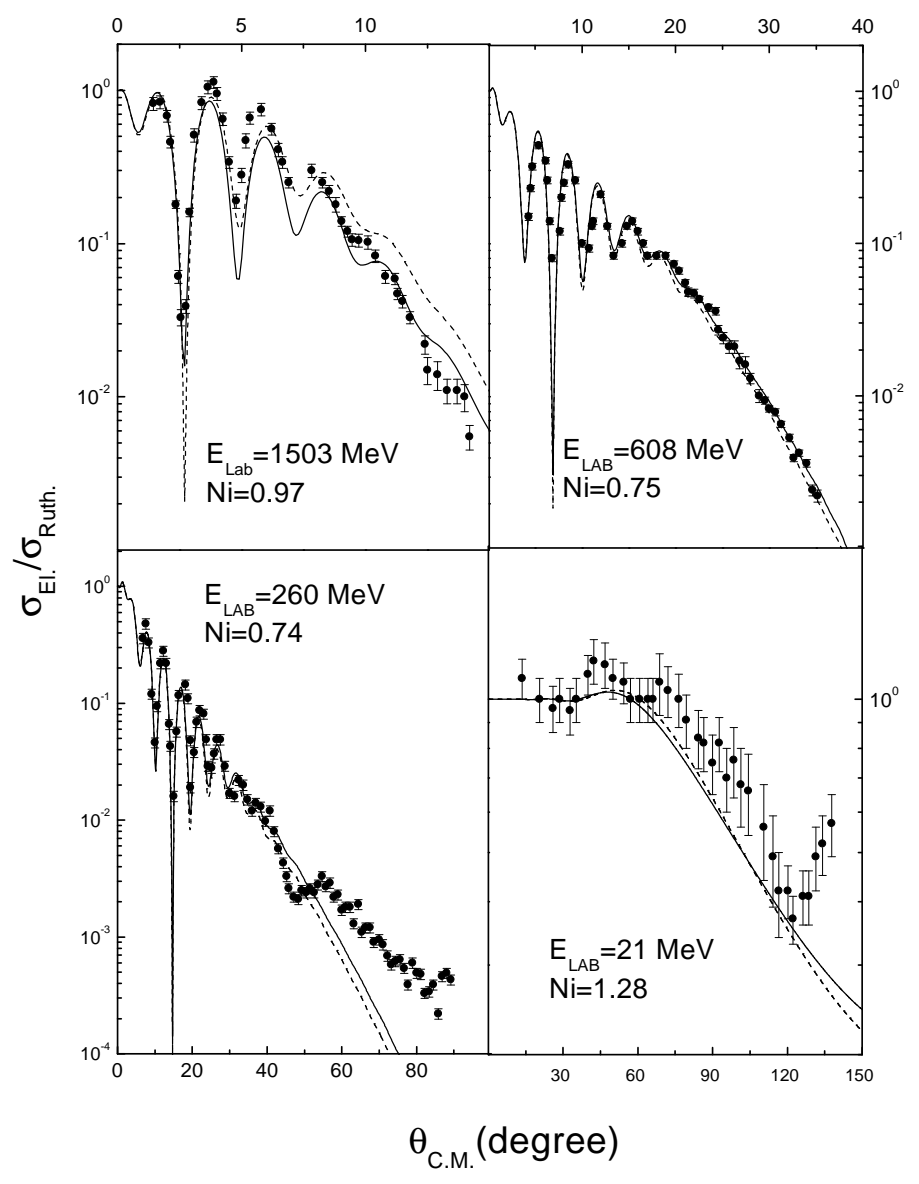

FIG. 2. The same of Fig. 1 for the ${ }^{16} \mathrm{O}+{ }^{12} \mathrm{C}$ system. 


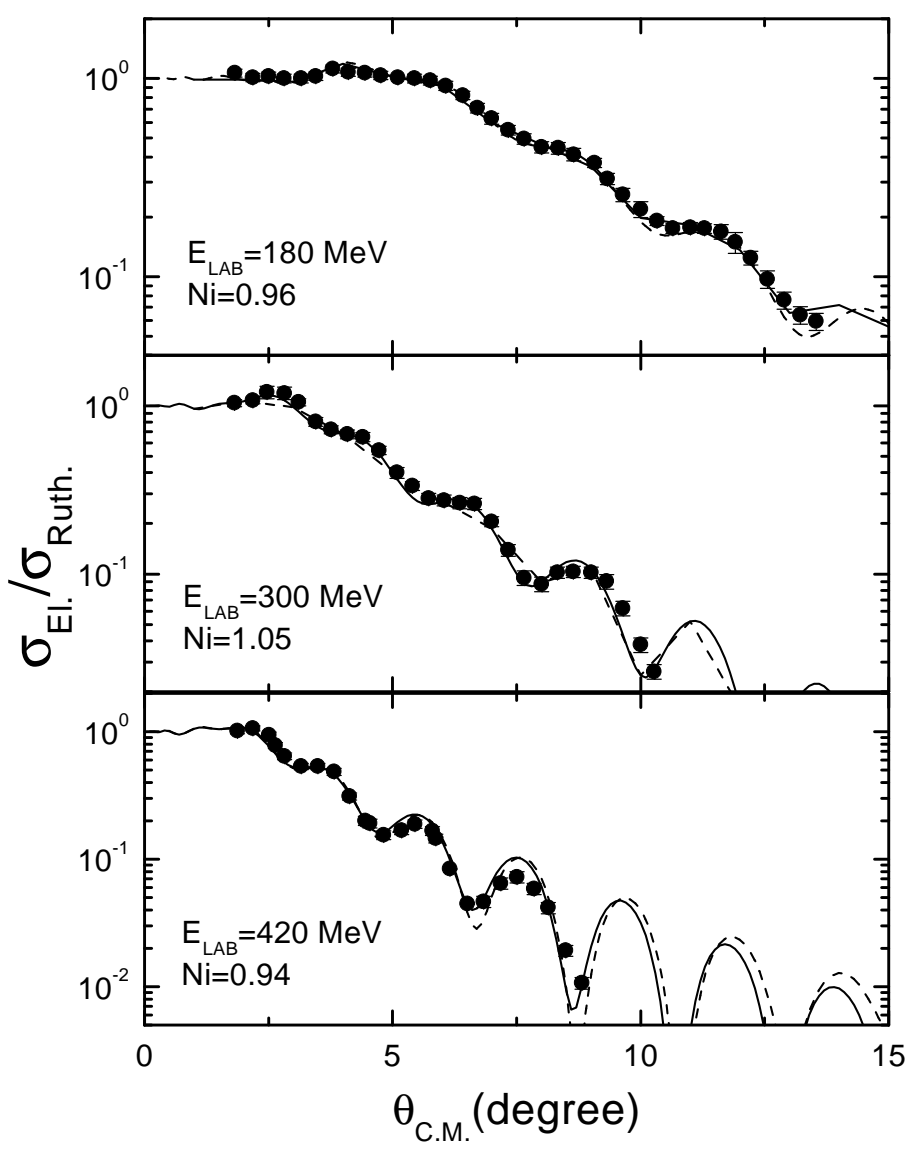

FIG. 3. The same of Fig. 1 for the ${ }^{12} \mathrm{C}+{ }^{40} \mathrm{Ca}$ system. 


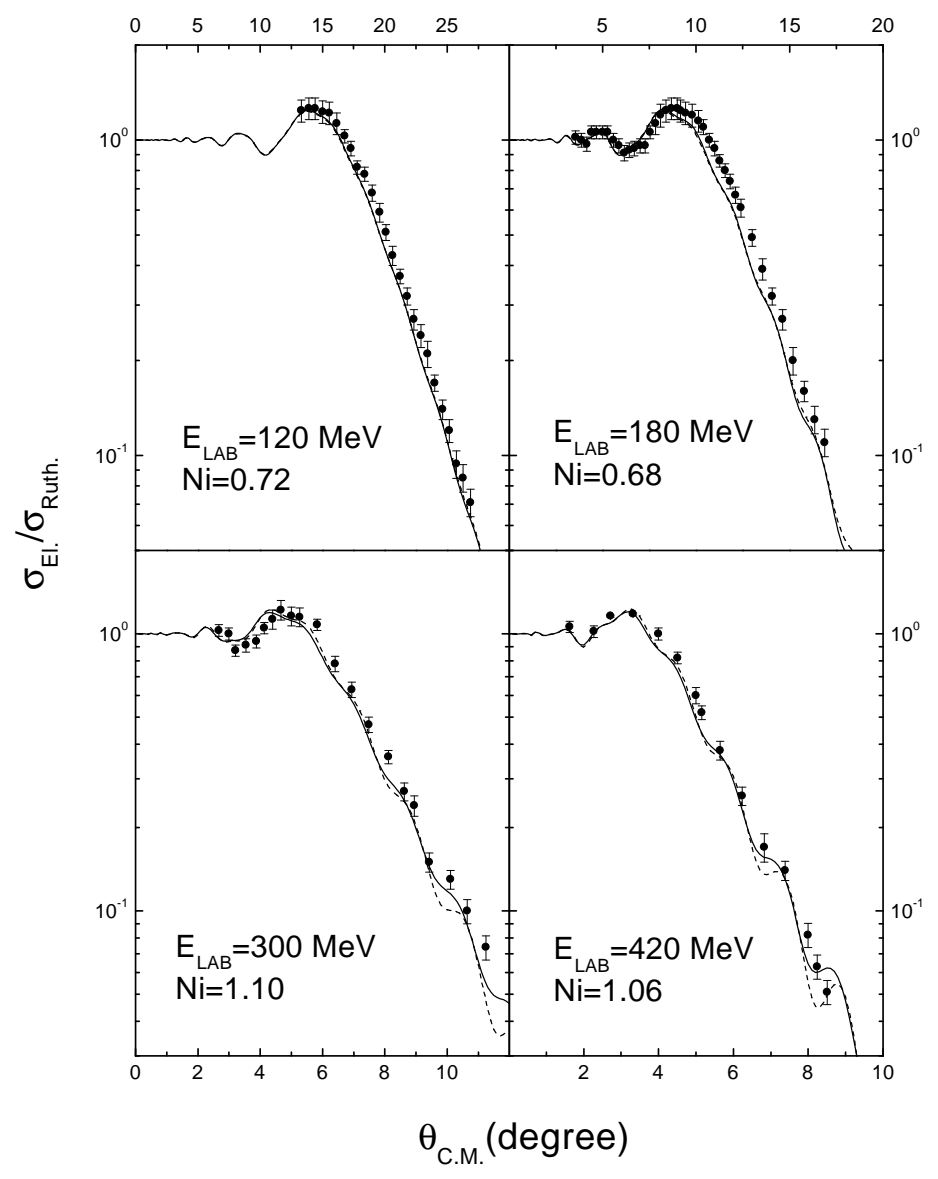

FIG. 4. The same of Fig. 1 for the ${ }^{12} \mathrm{C}+{ }^{90} \mathrm{Zr}$ system. 


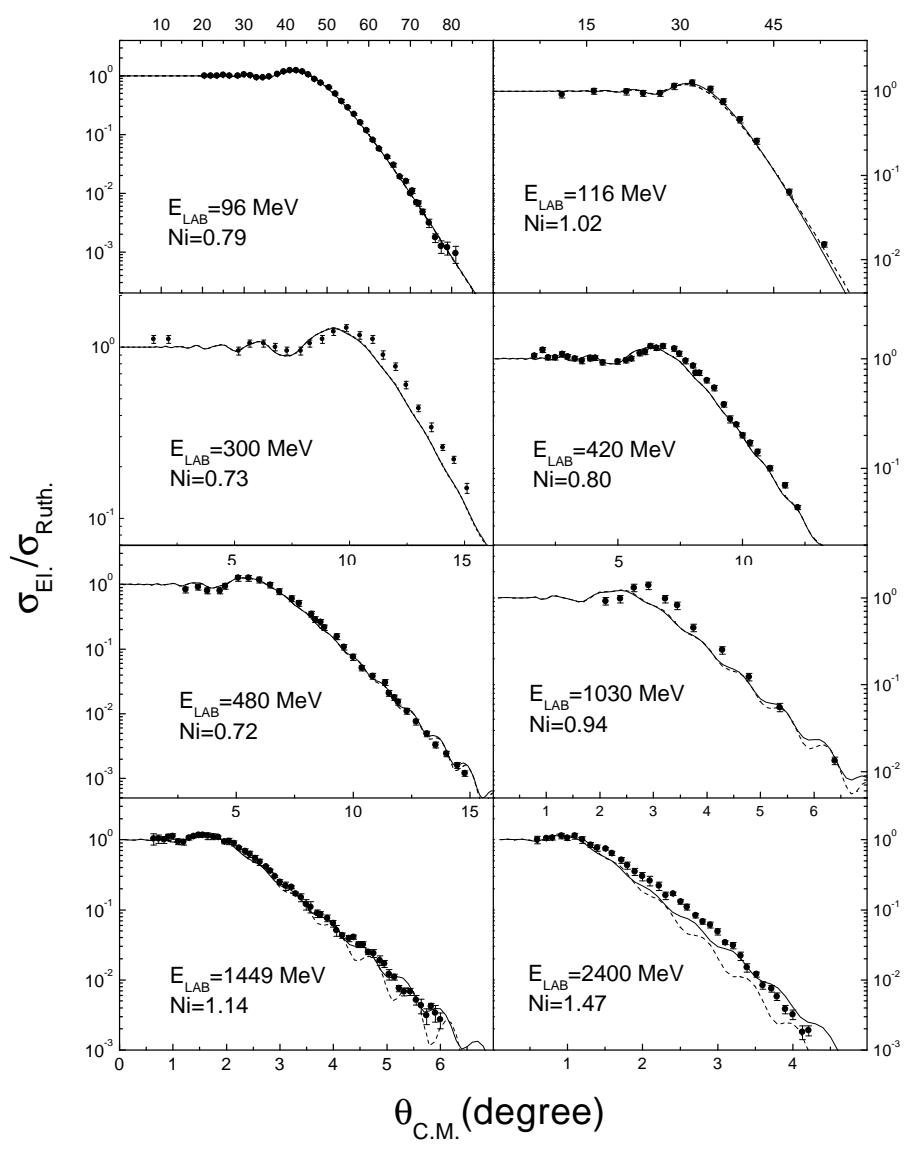

FIG. 5. The same of Fig. 1 for the ${ }^{12} \mathrm{C}+{ }^{208} \mathrm{~Pb}$ system. 


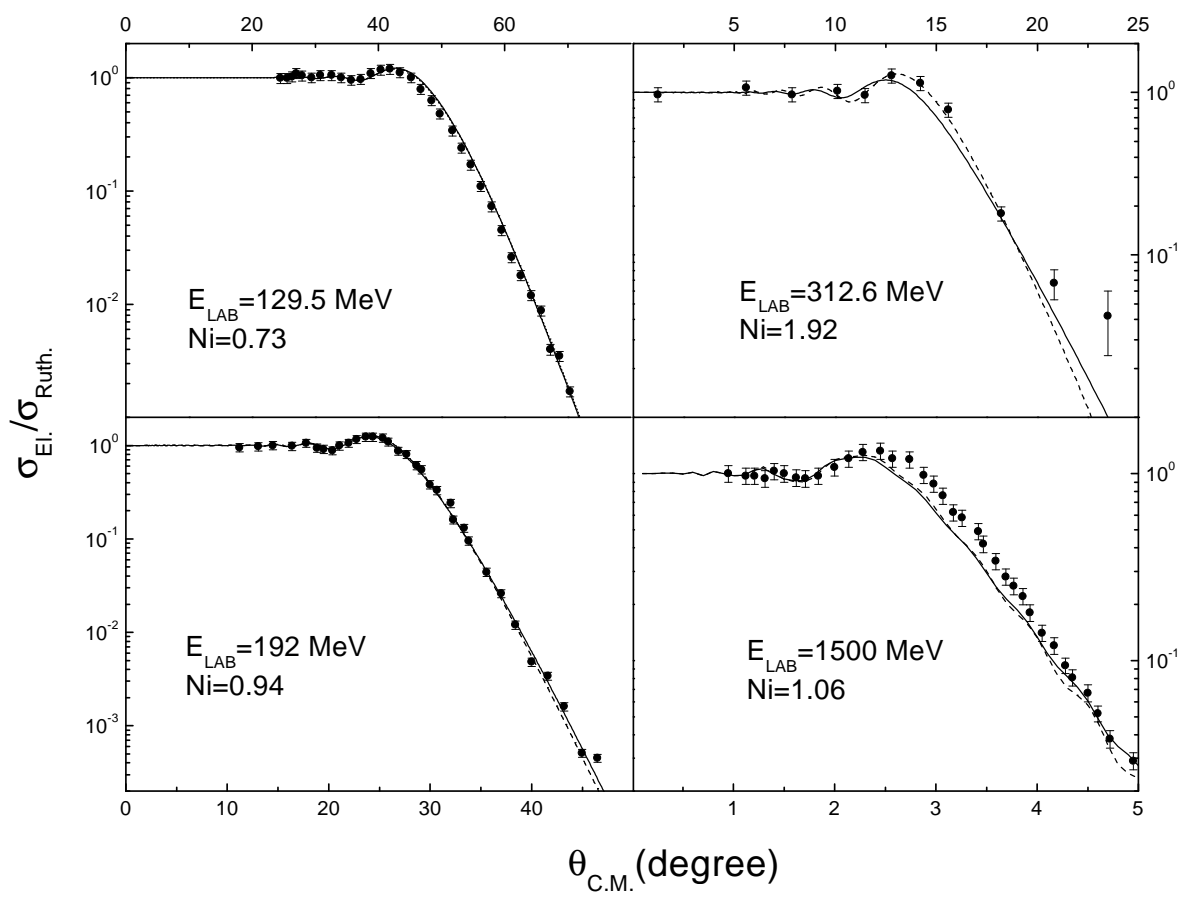

FIG. 6. The same of Fig. 1 for the ${ }^{16} \mathrm{O}+{ }^{208} \mathrm{~Pb}$ system. 


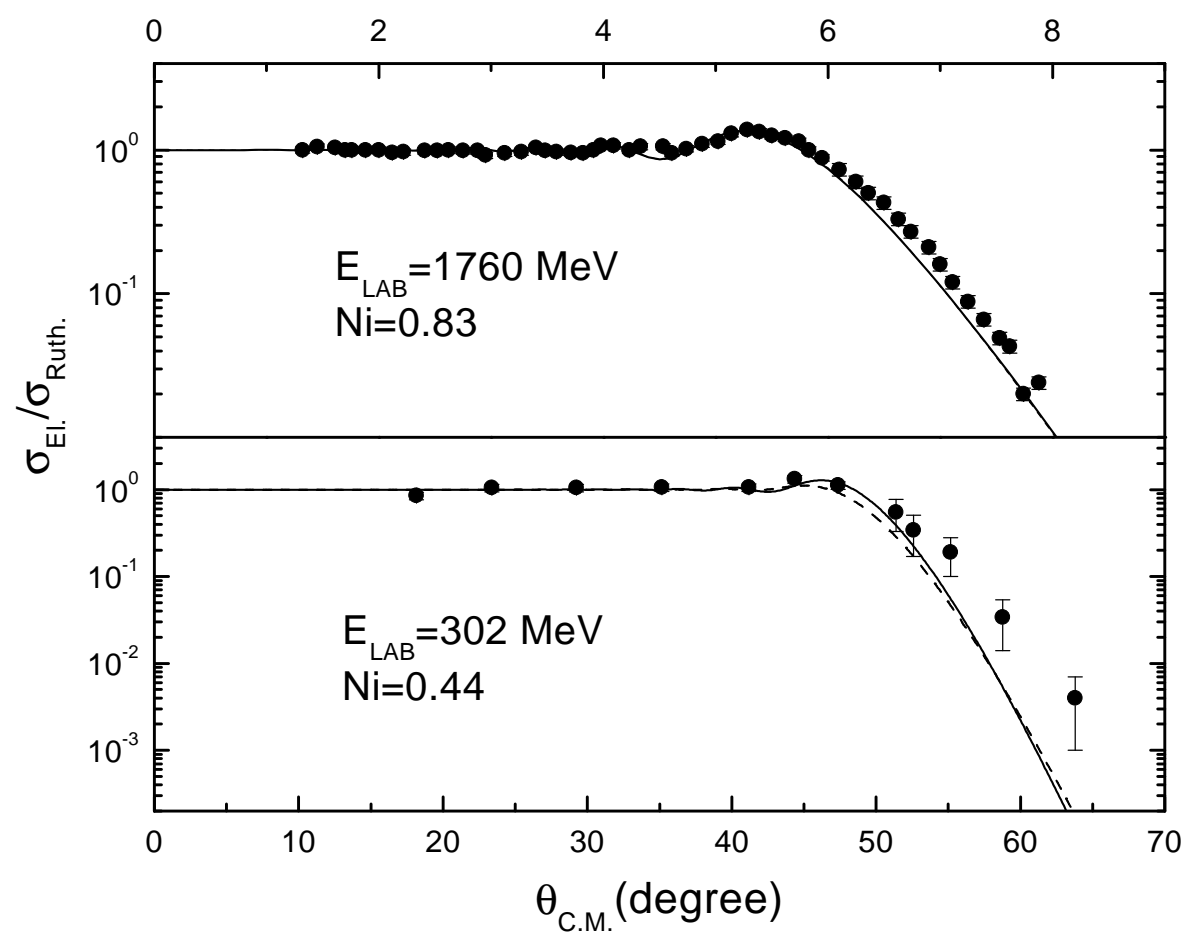

FIG. 7. The same of Fig. 1 for the ${ }^{40} \mathrm{Ar}+{ }^{208} \mathrm{~Pb}$ system. 


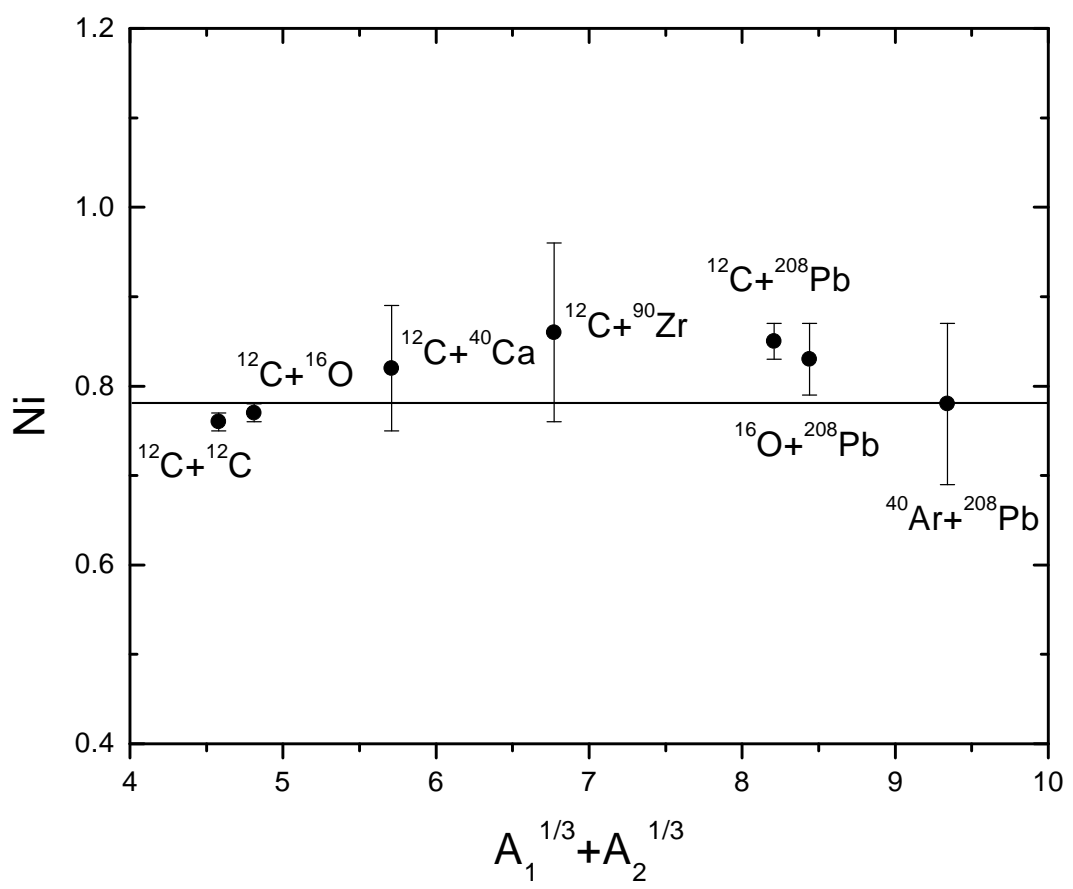

FIG. 8. The $N_{i}$ values for different systems obtained by adjusting the corresponding elastic scattering angular distributions. The solid line represents the average value $N_{i}=0.78$. 